\title{
Perceptually Motivated Shape Evolution with Shape-Preserving Property
}

\author{
Sergej Lewin, Xiaoyi Jiang, and Achim Clausing \\ Department of Mathematics and Computer Science, University of Münster \\ Einsteinstrasse 62, D-48149 Münster, Germany \\ \{slewin, xjiang, cl\}@math. uni-muenster.de
}

\begin{abstract}
In this paper we introduce a novel concept of shape evolution. It is perceptually motivated and takes the local shape symmetry into account. In particular, during the new shape evolution the shape parts in accordance with the human perception are removed step by step and the coarse parts remain geometrically unchanged during removing the fine parts. This shape-preserving property cannot be achieved by the popular Gaussian smoothing (evolution based on geometric heat flow) and related variants. Experimental results demonstrate the behavior and power of this new shape evolution scheme.
\end{abstract}

Keywords: Shape evolution, Shape perception.

\section{Introduction}

Research in psychology has shown that shapes are perceived by their parts [11]. The different shape parts have different importance for the perception of the complete shape, as illustrated by the following simple example. When considering a human's shape, we can say that the torso has greater importance for the perception than the arms. Moreover, the arms are more important than the hands and so on. This perception chain can be further continued and is called the perceptional hierarchy. Such a perceptual hierarchy can be represented by a perceptual tree, where the main part of a shape is the root of the tree. For a lot of shape classes this observation applies and leads to the idea of multi-scale shape representations.

The multi-scale representation can be computed by using a shape evolution. The shape evolution is a deformation process, which should be causal, in the sense that it maintains a hierarchical structure of geometric and perceptual features without introducing any new ones. There are different shape evolution approaches. For instance, Mokhtarian and Bober use the Gaussian smoothing to get the curvature scale space images [9]. Another interesting shape evolution named discrete curve evolution was proposed by Latecki and Lakämper [7. Other shape evolution approaches can be found in [48].

In Section 2 we introduce some principles for shape evolution. Then, we discuss the psychological observation about shape parts perception in Section 3. The new shape evolution approach and implementation details are given in Sections 4 and 5 . 
After that we present the experimental results and discuss the property of our new shape evolution approach in Section 6. Further discussion and future work will be given in Section 7 to conclude the paper.

\section{Shape Evolution Principles}

The main idea of shape evolution is the simplification of shapes. This shape simplification can be reached by gradually removing the shape details, whereas the less important details are removed firstly. Consequently, the shape evolution produces a hierarchy of shape parts. In addition such a gradual removing scheme enables to recognize the structure of coarse shape parts.

In the following we will use the boundary representation of shapes. In this case shape evolution can be implemented by successively deforming the curve. Without loss of generality we assume the curve is twice differentiable. Furthermore, we consider closed and simple initial curves only and require that the curve remain closed and simple during the evolution process.

As mentioned above, the shape evolution should be a fine-to-coarse hierarchical deformation process. From the perceptional point of view it is meaningful and important to introduce two general principles to guide the shape evolution process:

- During the evolution the curve should be simplified by incrementally removing details which correspond to the shape parts perceived by human beings. What we mean by human perception will be explained in the next section.

- Removing fine details during the evolution should not deform the coarse details geometrically.

In the remainder of this paper we will develop a novel shape evolution method by following these general principles.

\section{$3 \quad$ Perception of Shape Parts}

Research in psychology has shown that shapes are perceived by their parts [1. Accordingly, shape similarity strongly depends on the shape parts. Thus, it is one of the vital tasks in shape analysis to find the features or rules which the human uses to determine the shape parts or to decompose shapes into parts. In particular, it is important to find natural features for the cuts, which are straight lines with two end points on the shape boundary contour and separate the shape parts from the rest of the shape.

Psychological tests and observations have shown that three features are mainly used by the humans to determine the cuts, if there is no a priori knowledge about the shape. The first important feature is the negative curvature minima on the shape boundary contour. At least one of the cut end points should be such a negative curvature minimum. This so-called minima rule was proposed 


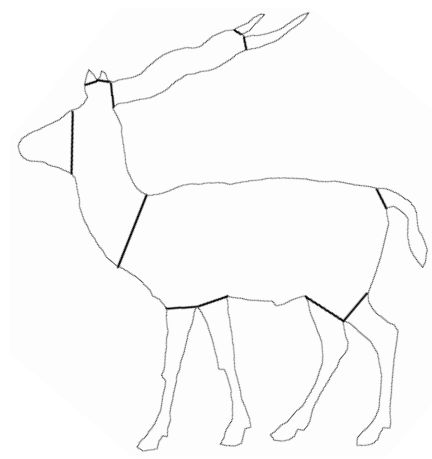

Fig. 1. Possible shape decomposition with cuts

by Hoffman and Richards 3 . Note that this rule does not specify which contour point should be the second one to be connected for one cut.

The second feature is the so-called short-cut rule [12. The cut should be as short as possible. But there are cases where longer cuts conform to human intuition more than shorter ones. In this case the local symmetry plays a large role for the cuts and is then used as the last feature for shape decomposition [11. Figure 1 shows the cuts which separate the shape into parts.

\section{New Shape Evolution Approach}

As mentioned above, a shape can be represented by a closed boundary curve $C:[0, L] \rightarrow \mathbb{R}^{2}$. Accordingly, the shape evolution can be described generally as a sequence of deformed curves $\left(C_{t}\right)_{t \geq 0}$ with $C_{0}=C$ and the deformation process can be described by the differential equation:

$$
\begin{array}{r}
C_{t}(s)=(x(s, t), y(s, t)) \\
\frac{\partial C}{\partial t}=V_{N} \boldsymbol{N}+V_{T} \boldsymbol{T}
\end{array}
$$

where $s$ is the arc length parameter, $t$ is the evolution time, $\boldsymbol{N}$ and $\boldsymbol{T}$ are the outward normal and tangent, respectively. During the evolution the curve is moving along its normal and tangential direction with the velocity $V_{N}$ and $V_{T}$, respectively. Since the motion along the tangential direction merely affects the curve parameterization [6], the differential equation of evolution can be converted into a differential equation with motion along the normal direction only by changing the parametrization:

$$
\frac{\partial C}{\partial t}=V_{N} \boldsymbol{N}
$$

The different shape evolution processes known from the literature arise from different choices of the velocity function $V_{N}$. The most common choices are the constant function, the curvature function with negative sign and the linear 
combination of these functions [2519. These choices correspond to the following differential equations, respectively:

$$
\begin{aligned}
& \frac{\partial C}{\partial t}=\boldsymbol{N} \\
& \frac{\partial C}{\partial t}=-\kappa \boldsymbol{N} \\
& \frac{\partial C}{\partial t}=\left(\beta_{0}-\beta_{1} \kappa\right) \boldsymbol{N}
\end{aligned}
$$

where $\kappa$ represents the curvature at a shape point. The sequence of curves obtained with the constant velocity (Eq. 1) is referred to as offsets [6]. In this case the shape curve does not retain simple during the evolution. In the case of the linear combination of the constant and curvature velocity (Eq. 3) the parameter $\beta_{0}$ and $\beta_{1}$ control the behavior of curves during the evolution. However, finding the appropriate parameter values is a difficult task.

In the following we turn to the more interesting case with the velocity equals to the curvature (Eq. 2). In this case the curve points with the negative curvature move outwards and the curve point with positive curvature move inwards. Unfortunately, this evolution scheme contradicts the evolution principles discussed before. The complete shape is deformed in each evolution step, leading to both the removal of fine details from the shape and geometric deformation of coarse details simultaneously, as can be seen in Figure 2. Thus, we pay the price of blowing up the more coarse details for the removal of fine details, which is clearly undesired.

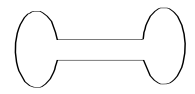

(a)

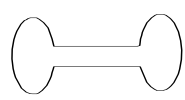

(f)

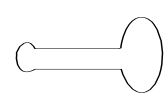

(b)

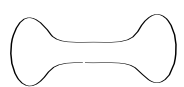

(g)

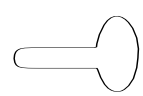

(c)

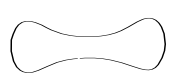

(h)

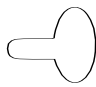

(d)

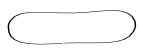

(i) (e)
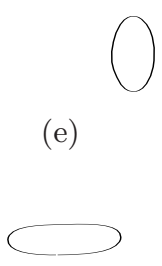

(j)

Fig. 2. Shape evolution with our approach (upper row) and with Gaussian smoothing (lower row)

To avoid these disadvantages we propose to introduce the following changes in the curvature controlled shape evolution. The deformation of complete shape curve should be replaced by deformation of only curve segments. The selection of such segments on the curve should take the local symmetry into consideration. Furthermore, shape points with positive curvature should be smoothed only in order to avoid the blowing-up-effect.

Since the fine details are removed first during the evolution, the shape segment to deform should be a part of the finest shape detail. We propose to partition 
the curve in convex and concave segments firstly, which can be described by partitioning the parameterization interval in subintervals according to the convex and concave segments. Because only the points with positive curvature should be smoothed, the curve segment to deform can thus be the part of one convex segment only. Apparently, the curvature is a good measure for fineness of the shape parts and we should consider the convex segment with maximal curvature point for the next deformation operation.

The maximal curvature point lies on the boundary of the finest shape part and can be considered as the symmetry point. Around this point the segment to deform can be selected in the following way: Let the convex segment with maximal curvature point be represented by subinterval $(b, e)$ of the parametrization interval, where $b$ and $e$ are the inflection points, and $p_{m} \in(b, e)$ the position of the point with maximal curvature. Obviously, $p_{m}$ decomposes the segment $(b, e)$ in two halves $\left(b, p_{m}\right)$ and $\left(p_{m}, e\right)$. If the half segment $\left(b, p_{m}\right)$ is longer than the half segment $\left(p_{m}, e\right)$ (The other case is handled similarly), we shorten the segment $\left(b, p_{m}\right)$ to $\left(s, p_{m}\right)$ so that the lengths of $\left(s, p_{m}\right)$ and $\left(p_{m}, e\right)$ are equal. The segment to deform is then $(s, e)$. This convex segment shortening takes the local shape symmetry into account.

We denote the selected segment to deform at evolution time $t$ as maxseg $(t)$. Now a new velocity function for shape evolution can be defined by:

$$
V_{N}(s, t)= \begin{cases}-\kappa(s, t) ; & \text { if } s \in \operatorname{maxseg}(t) \\ 0 ; & \text { otherwise }\end{cases}
$$

Figure 3 shows the new evolution process, which ends with a convex shape.

\section{Implementation Details}

The new shape evolution approach can be simply implemented by means of the Gaussian smoothing. The shape evolution with curvature velocity is referred to as geometric heat flow, which corresponds to Gaussian smoothing of the curve [10. Especially, the arc length evolution based on Gaussian smoothing can be expressed as follows:

$$
\begin{aligned}
& C_{\sigma}(\Psi)=\left(x_{\sigma}(\Psi), y_{\sigma}(\Psi)\right), \sigma \geq 0 \\
& x_{\sigma}(\Psi)=x(\Psi) \otimes g(\Psi, \sigma), \\
& y_{\sigma}(\Psi)=y(\Psi) \otimes g(\Psi, \sigma)
\end{aligned}
$$

where $\Psi\left(w, \sigma_{0}\right)$ with any $\sigma_{0} \geq 0$ is a continuous and monotonic function of $w$ and $g(u, \sigma)=\frac{1}{\sigma \sqrt{2 \pi}} e^{-u^{2} / 2 \sigma^{2}}$ is the Gaussian kernel function. The value of $\sigma$ determines the degree of smoothing. $\Psi$ remains the normalized arc length parameter as the curve evolves [9]. Generally, it is difficult to compute the function $\Psi$ analytically. In the discrete case, however, this arc length evolution can be computed by a resampling technique. Firstly, the Gaussian smoothing based on a small $\sigma(=1.0)$ value is performed. Then the resulting curve is resampled with a constant sampling rate. These two steps are repeated to achieve a smoothing of arbitrary $\sigma$ value 9 . 
This Gaussian smoothing with resampling technique can be adapted for the new shape evolution as follows. To reduce the noise influence on curvature computation an initial curve is generally smoothed by a small $\sigma=1.0$. Then, we perform the following steps:

1. The curvature is computed for each curve point.

2. The curve is decomposed in concave (negative curvature $\kappa)$ and convex $(\kappa \geq$ 0 ) segments and the segment with maximal curvature point is chosen.

3. The chosen segment is shortened symmetrically so that the number of sample points on the left and right of the maximal curvature point is equal.

4. The Gaussian smoothing based on $\sigma=1.0$ is applied to the shortened segment.

5. The smoothed segment is resampled and the curvature for the segment points recomputed.

The steps $2-5$ are repeated until the shape curve becomes convex.

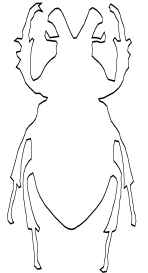

(a)

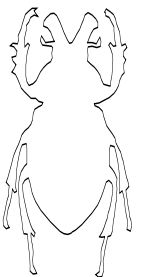

(i)

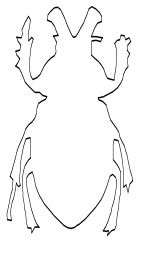

(b)

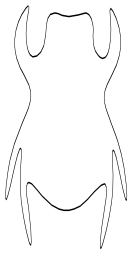

(j)

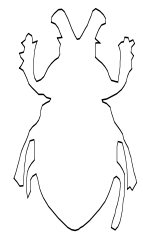

(c)

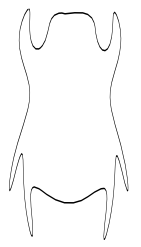

(k)

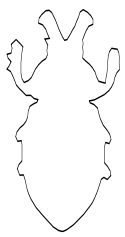

(d)

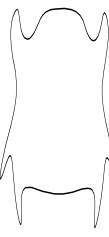

(1)

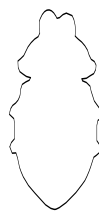

(e)

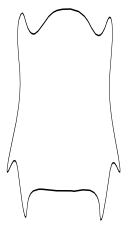

(m)

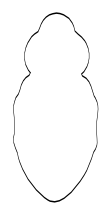

(f)

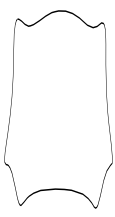

(n)

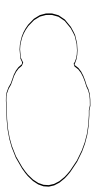

(g)

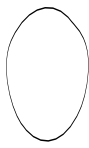

(h)

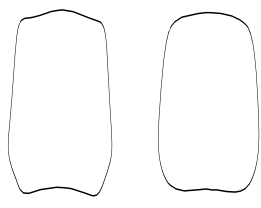

(o)

(p)

Fig. 3. Shape evolution with our approach (upper row) and with Gaussian smoothing (lower row)

\section{Properties of New Shape Evolution Approach}

The new shape evolution approach has some interesting properties. It can be shown that the shape evolution process will converge to a convex shape. At latest then the evolution process can be stopped. Figures 24 show this convergence property.

The most interesting property is that the coarse shape parts remain geometrically unchanged while the fine parts are removed. In addition the removed parts 
accord with the human perceived shape parts well. This can be seen in Figures24 Note that this shape-preserving property can also been achieved to some degree by the methods presented in 48 . The evolution process there is controlled by an adaptive, yet global velocity function based on a shape width concept so that a shape preservation cannot be fully guaranteed in contrast to our work.

To compare the Gaussian smoothing (corresponding to the evolution with the curvature velocity) with our shape evolution we consider the example in Figure 3. It shows two sequences of beetle shapes produced by Gaussian smoothing (lower row) and our approach (upper row). Obviously, the beetle's main body consists of three parts. In our shape evolution one easily recognizes the structure of the body, see Figure 3 (e) and (f). In contrast the body structure is lost during the Gaussian smoothing and thus cannot be recognized in the evolution process.

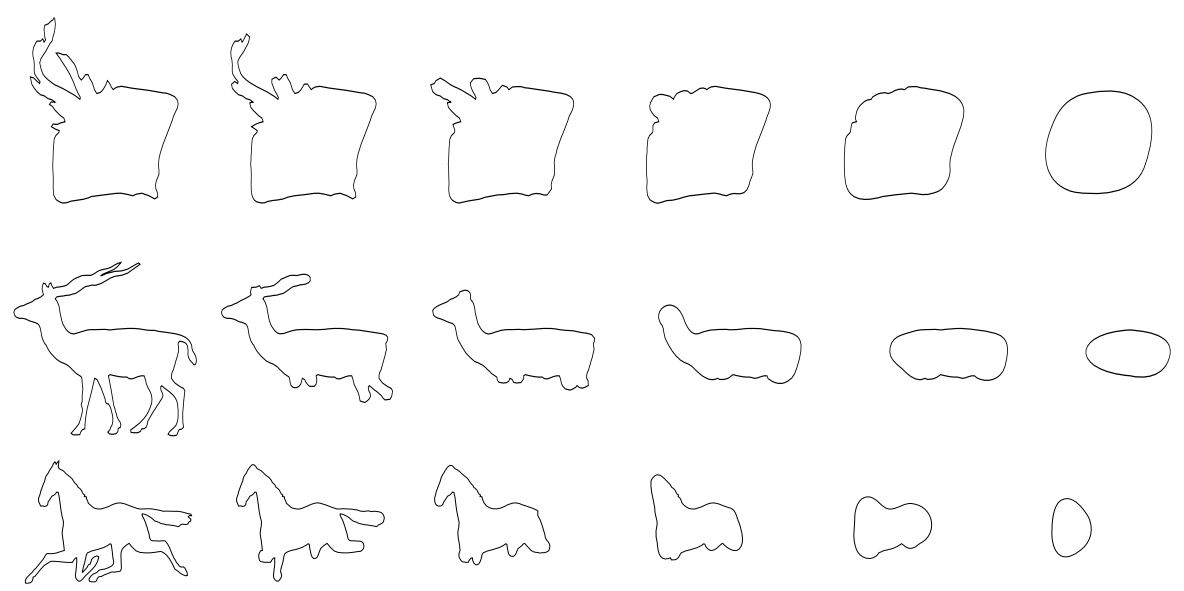

Fig. 4. New shape evolution approach

Our approach is robust to some kind of noise. In the most cases the noise accords with the points on the shape boundary with high curvature value. Since our approach removes the segments with high curvature firstly, the noise is removed from the shape at the begin of the evolution process.

\section{Conclusions and Future Work}

In this paper we have introduced a novel concept of perceptually motivated shape evolution. It can be easily implemented based on the Gaussian smoothing. Our approach has the interesting and important properties of convergence and shape-preservation. In particular, the shape-preserving property cannot be achieved by the popular Gaussian smoothing and related variants whose behavior is controlled by the local curvature alone, but not the more global perceptual features. This unique property enables us to explore the part structure of shapes in the context of part hierarchy. 
Our work can be extended in various directions. The new shape evolution can be used to decompose a shape into shape parts which accord with the human shape perception. Furthermore, we believe that the new evolution can be used to compute the shape skeleton robustly. Shape retrieval is another topic worth of investigation.

\section{References}

1. Bierderman, I.: Recognition-by-Components: A Theory of Human Image Understanding. Psychological Review 94(2), 115-147 (1987)

2. Blum, H.: A transformation for extracting new descriptors of form. In: WhatenDunn, W. (ed.) Models for the Perception of Speech and Visual Form., pp. 362-380. MIT Press, Cambridge (1967)

3. Hoffman, D.D., Richards, W.: Parts of Recognition. Technical Report AIM-732 (1983)

4. Jiang, X., Lewin, S.: An Approach to Perceptual Shape Matching. In: Bres, S., Laurini, R. (eds.) VISUAL 2005. LNCS, vol. 3736, pp. 109-120. Springer, Heidelberg (2006)

5. Kimia, B., Tannebaum, A., Zucker, S.: Shapes, shocks, and deformations I: the components of two-dimensional shape and the reaction-diffusion space. IJCV 15, 189-224 (1995)

6. Kimmel, R.: Numerical Geometry of Images: Theory, Algorithms and Applications. Springer, Heidelberg (2004)

7. Latecki, L.J., Lakämper, R.: Convexity rule for shape decomposition based on discrete contour evolution. Computer Vision and Image Understanding 73, 441454 (1999)

8. Lewin, S., Jiang, X., Clausing, A.: Shape Evolution Driven by a Perceptually Motivated Measure. In: Bebis, G., Boyle, R., Parvin, B., Koracin, D., Paragios, N., Tanveer, S.-M., Ju, T., Liu, Z., Coquillart, S., Cruz-Neira, C., Müller, T., Malzbender, T. (eds.) ISVC 2007, Part II. LNCS, vol. 4842, pp. 214-223. Springer, Heidelberg (2007)

9. Mokhtarian, F., Bober, M.: Curvature Scale Space Representation: Theory, Applications \& MPEG-7 Standardisation. Kluwer Academic, Dordrecht (2003)

10. Sapiro, G.: Geometric Partial Differential Equations and Image Analysis. Cambridge University Press, Cambridge (2001)

11. Siddiqi, K., Kimia, B.B.: Parts of Visual Form: Computational Aspects. IEEE Transactions on Pattern Analysis and Machine Intelligence 17(3), 239-251 (1995)

12. Singh, M., Seyranian, G., Hoffman, D.: Parsing silhouettes: The short-cut rule. Perception and Psychophysics 61, 636-660 (1999) 